\title{
EDITORIAI.
}

\section{The Hand in Liprosy}

The loss of manual skill from whatever cause is a most serious disability. These contributions of Paul Brand and Miss Ruth Thomas are important because they offer a real opportunity to prevent or reclaim the disabled hand resulting from leprosy. They mark a stage in the amelioration of this disease.

One of the major problems facing the orthopaedic surgeon is the restoration of the patient disabled from poliomyelitis. This disease presents many similar problems to those faced in leprosy. Both are infective and neither infection can as yet be controlled, though there is much to lead us to think that this may not be too far away. Until this fundamental solution has been reached, the orthopaedic surgeon can offer amelioration of the disabilities, often in striking fashion.

The patient crippled by poliomyelitis was once shunned and left to his own resources. At the turn of the century, and even earlier, Hugh Owen Thomas and Robert Jones and others developed operative procedures which brought relief to the untreated, terribly deformed paralytic, but their results were limited by the severity of the deformities with which they dealt. Important in their time, these operations are now seldom performed; we now rarely allow such deformities to develop. We have learned that in motor paralysis, deformity is caused by disuse and muscle imbalance. Deformity is preventable by maintaining a full range of joint motion, active or passive and the balancing of muscle pull by tendon transfer.

In poliomyelitis the crippled hand was the last to succumb to the surgeons attack. In the hand, stiffness and contracture are quickly the penalty of omission, nowhere in the body is it more obvious that maintenance of function, not restoration of function is the real solution to our problems.

Miss Thomas has shewn that this aphorism is no less true of leprosy than of poliomyelitis. She demonstrates that deformity of the hand in leprosy is unnecessary and the result of failure to maintain movement. Deformity is preventable and unnecessary. This, she rightly stresses, is not achieved easily or quickly, enthusiasm and never ceasing vigilance, are, and will always be essential. Muscle strength is difficult to assess accurately and the electrical reactions as used by Miss Thomas are often quantitatively unreliable. May we commend the now generally accepted M.R.C. method of grading voluntary power?

Paul Brand has pioneered but unlike some pioneers he has not become unbalanced. Applying the established principles of hand reconstruction in poliomyelitis and peripheral nerve injury to the 
fundamentally similar problem of leprosy, he has achieved notable success. This, despite the difficulty presented by the contracted and stiff hands which have been his material.

From our now well established knowledge of hand paralysis arising from other causes, it is certain that Brand and Miss Thomas based their practice on sound orthopaedic principles. In future active exercises and the maintenance of joint range by passive movements can avoid contracture and stiffness. Surgical reconstruction of the supple mobile hand suffering from muscle imbalance in the intrinsic and thenar groups of muscles will be even more worthwhile than it is in poliomyelitis, where the long flexors and extensors are so frequently involved.

Little has been said of the sensory loss although in many ways this is the more serious disability in leprosy. The reason for neglecting this aspect is a simple one, it is not amenable to surgical cure. The solution to this must be in prevention or cure of the disease in its earliest stage.

J. I. P. JAMES.

\section{APPRECIATION AND CONGRATULATIONS}

The Editor is desirous of expressing his thanks to the contributors to this number of the Review, which to him marks a landmark in leprosy history. How vividly does he bring to mind the half halting and ineffective attempts to improve the lot of the deformed patient before the orthopaedic surgeon and the physiotherapist were persuaded that leprosy was a disease worthy of their attention. It will be seen that the appeal of those who technically may no longer have leprosy, but whose constant cry is " What can we do with these hands? " has been fully, skillfully and altogether admirably answered by $\mathrm{Mr}$. Brand and his enthusiastic team at Vellore.

We would especially thank Mr. James not only for his able summary of the position of orthopaedic surgery and physiotherapy in leprosy, but for his constant willingness to give of his skill and time to leprosy patients in Great Britain, and we would also express our appreciation for the co-operation of his staff.

To Sir Leonard Rogers we would offer our heartiest congratulations on reaching his 87 th year. There is no one in the history of Tropical Medicine who has made a greater impact. When leprosy was fully encompassed by the words 'Leper' and 'Unclean' he stepped forth, a determined warrior, and said " These things shall not be ". Readers will realise on reading Sir Leonard Rogers' contribution how far his vision, his wise counselling and his dynamic personality have hastened this day, when we can say men and women, and, above all, little children, need no longer be gripped by paralysing fear. 